\title{
Circ-TOP2A acts as a ceRNA for miR-346 and contributes to glioma progression via the modulation of sushi domain-containing 2
}

\author{
JUNZHI SANG ${ }^{1}$, XING LI ${ }^{2}$, LUTING LV $^{3}$, CHUNYU ZHANG $^{1}$, XIAOQIAN ZHANG ${ }^{1}$ and GUOAN LI ${ }^{1}$ \\ Departments of ${ }^{1}$ Magnetic Resonance Imaging, ${ }^{2}$ Ultrasonics and ${ }^{3}$ Neurology, \\ The Second Affiliated Hospital of Qiqihar Medical University, Qiqihar, Heilongjiang 161000, P.R. China
}

Received July 22, 2020; Accepted January 18, 2021

DOI: $10.3892 / \mathrm{mmr} .2021 .11894$

\begin{abstract}
Dysregulated circular RNAs (circRNAs) are involved in the carcinogenesis and progression of multiple human malignancies. Knowledge of circRNAs in glioma (GM) is limited and further study to uncover new therapeutic targets for GM is urgently required. The present study demonstrated that circ-TOP2A was elevated in GM tissue specimens and cells and that circ-TOP2A levels indicated an unfavorable clinical prognosis in GM. Functionally, circ-TOP2A knockdown reduced viability, migration and invasion and triggered apoptosis in LN229 cells. Ectopic expression of circ-TOP2A aggravated these malignant behaviors in U87MG cells. In terms of mechanism, RNA-seq was performed to discover the potential targets regulated by circ-TOP2A. Circ-TOP2A acted as a competing endogenous RNA to upregulate sushi domain-containing 2 (SUSD2) expression by sponging microRNA (miR) 346. Rescue assays revealed that the oncogenic function of circ-TOP2A was partially dependent on its regulation of the miR-346/SUSD2 axis. In conclusion, the present study identified that circ-TOP2A promoted GM proliferation and aggressiveness via miR-346/SUSD2 signaling, which is a potential prognostic biomarker and therapeutic target for GM.
\end{abstract}

\section{Introduction}

Glioma (GM) is the commonest type of primary brain tumor (1). Based on the histopathologic classification formulated by the World Health Organization, glioma can be graded into four phases (I-IV) (2). The overall survival rate of GM patients is

Correspondence to: Dr Junzhi Sang, Department of Magnetic Resonance Imaging, The Second Affiliated Hospital of Qiqihar Medical University, 37 Zhonghuaxi Road, Qiqihar, Heilongjiang 161000, P.R. China

E-mail: yuye912rr@aliyun.com

Key words: glioma, circ-TOP2A, micro RNA 346, sushi domain-containing 2 only $\sim 14$ months (3). Although novel therapies against GM have been applied, the situation is still grim for patients (4). At present, the pathogenesis of GM remains elusive.

Circular RNAs (circRNAs) are characterized by a closed-loop structure with limited protein coding capacity (5). The majority of these covalently closed loop circRNAs are produced with exons via the head-to-tail junction (6). Previously, they were believed to be functionless owing to errors in splicing (7). With the development of high-throughput RNA sequencing, they were identified as important factors in numerous biological processes (8). They are relatively stable and resistant to ribonuclease $\mathrm{R}$ digestion, making them better biomarkers for diagnosis and treatment than linear RNAs (9). Accumulating evidence suggests that circRNAs can be regulators in the development and progression of cancers $(10,11)$. Nevertheless, the effects and molecular mechanisms of circRNAs in GM are not fully understood and thus need to be extensively and systemically investigated.

Previously, Wang et al (12) performed circRNA sequencing and identified several dysregulated circRNAs in GM tissues relative to their normal counterparts. The present study aimed to identify the potential therapeutic target for GM from the view of circRNAs.

\section{Materials and methods}

Clinical specimens. A total of 74 pairs of $\mathrm{GM} / \mathrm{matched}$ noncancerous specimens (Table SI) were harvested from patients at the Second Affiliated Hospital of Qiqihar Medical University, Heilongjiang, China, between January 2013 and January 2015. A validation cohort consisting of 74 GM patients (age range: 23-71 years old; 49 male, 25 female) was selected according to the following inclusion criteria: i) Patients were diagnosed with GM by preoperative imaging examination (Fig. 1A) and postoperative pathology; ii) patients who underwent radical resection with a clear surgical margin; iii) patients with available follow-up information; iv) patients with a survival time of more than 1 month; v) none of the patients received anticancer treatment before the surgery; and vi) patients who had no history of other malignancies. Exclusion criteria were patients with serious diseases or severe chronic diseases, such as cardiovascular and cerebrovascular 
diseases. The research was authorized by the Ethics Committee of our hospital (approval number: KY2020-012) and written informed consent was acquired from each patient.

Cell lines and transfection. LN229, U251 and U87MG (cat. no. TCHu138, glioblastoma of unknown origin) were provided by the Chinese Academy of Sciences and normal human astrocytes (NHA; cat. no. CC-2565) were acquired from Lonza Group Ltd. All the cells were cultured in RPMI-1640 (Gibco; Thermo Fisher Scientific, Inc.) with $10 \%$ fetal bovine serum (FBS, Invitrogen; Thermo Fisher Scientific, Inc.) in a cell incubator $\left(37^{\circ} \mathrm{C}, 5 \% \mathrm{CO}_{2}\right)$. The sequences of circ-TOP2A and SUSD2 were synthesized and cloned into the pcDNA 3.1 circRNA mini vector and pcDNA 3.1 vector, respectively. Short interfering (si)RNAs specific against circ-TOP2A and SUSD2, miR-346 mimics and inhibitors were purchased from GenePharma (Shanghai, China). The plasmids and small RNAs were transfected using Lipofectamine ${ }^{\circledR} 3000$ (Invitrogen; Thermo Fisher Scientific, Inc.) according to the manufacturer's protocol. Serum-free medium $(125 \mu \mathrm{l})$ was used to dilute $5 \mu \mathrm{l}$ of Lipofectamine ${ }^{\circledR} 3000$ in a $1.5 \mathrm{ml}$ EP tube. Meanwhile, $5 \mu 1$ of siRNA $(20 \mu \mathrm{M})$ or $2.5 \mu \mathrm{g}$ of plasmid vector with $5 \mu \mathrm{l}$ of $\mathrm{P} 3000^{\mathrm{TM}}$ reagent was diluted in $125 \mu \mathrm{l}$ serum-free medium. After five min of incubation at room temperature, the reagents in the two tubes were combined. After 15-20 min, the mixtures were added into a $2.5-\mathrm{cm}$ dish filled with serum-free medium. Following $8 \mathrm{~h}$ of incubation, the medium was replaced with medium containing 10\% FBS. Transfection was confirmed by reverse transcription-quantitative (RT-q)PCR at $48 \mathrm{~h}$ after transfection. The targeted sequences of the siRNAs specifically targeting circ-TOP2A were: si-circ-TOP2A-1, 5'-ATG CAACTCTATGACATGGAT-3' and si-circ-TOP2A-2, 5'-CAT GCAACTCTATGACATGGA-3'.

$R T$-qPCR. RT-qPCR was performed as previously described (13). Briefly, total RNA was extracted with the TRIzol $^{\circledR}$ (Thermo Fisher Scientific, Inc.) method according to routine RNA extraction procedures in the laboratory according to the manufacturer's protocol. The isolated RNA was reverse transcribed into cDNA (Roche Diagnostics) in accordance with the manufacturer's instructions. Then, RT-qPCR assay was conducted on a 7500 fast Real-Time PCR system (Applied Biosystems; Thermo Fisher Scientific, Inc.) using SYBR Green Master (Roche Diagnostics) according to the manufacturer's instructions. The reaction volume was $50 \mu \mathrm{l}$. The thermocycling conditions were as follows: $90^{\circ} \mathrm{C}$ for $5 \mathrm{~min}, 90^{\circ} \mathrm{C}$ for $15 \mathrm{sec}$ and $60^{\circ} \mathrm{C}$ for $30 \mathrm{sec}$ for 40 cycles. U6 and GAPDH were used as internal controls. PCR primers were as follows: circ-TOP2A, forward, 5'-GGCAGAGAGAGTTGG ACTACAC-3' and reverse, 5'-CTTCTCCATTGAAGGGCT TGAG-3'; U6, forward, 5'-ATTGGAACGATACAGAGAAGA TT-3' and reverse, 5'-GGAACGCTTCACGAATTTG-3' and GAPDH, forward 5'-GGGAGCCAAAAGGGTCAT-3' and reverse, 5'-GAGTCCTTCCACGATACCAA-3'. Each reaction was performed in triplicate and results were calculated using the $2^{-\Delta \Delta \mathrm{Cq}}$ method (14).

Actinomycin D treatment. A total of $2.5 \times 10^{5}$ cells were inoculated in 6-well plates and cultured for $48 \mathrm{~h}$ at $37^{\circ} \mathrm{C}$. Then, the transcriptional inhibitor actinomycin D (EMD Millipore) was added to the culture medium at $2 \mathrm{mg} / \mathrm{ml}$ for $0,4,8,12$ and $24 \mathrm{~h}$ at $37^{\circ} \mathrm{C}$. Then, total RNA was extracted, and RT-qPCR was used to detect the expression levels of circ-TOP2Aand TOP2A mRNA, as aforementioned.

Western blotting. Proteins were isolated using RIPA buffer (Beijing Solarbio Science \& Technology Co., Ltd.). The protein concentration was detected using a BCA detection kit (Beijing Solarbio Science \& Technology Co., Ltd.). Then, $30 \mu \mathrm{g}$ samples were separated by $10 \%$ SDS-PAGE and transferred onto PVDF membranes. After immersion in 5\% skimmed milk at $22-25^{\circ} \mathrm{C}$ for $2 \mathrm{~h}$, the membrane was incubated with anti-SUSD2 (1:2,000; cat. no. ab182147) and anti-GAPDH (1:10,000; cat. no. ab181602; both Abcam) primary antibodies overnight at $4^{\circ} \mathrm{C}$. After incubation with the HRP-conjugated secondary antibody (1:5,000; cat. no. ZB-2306; OriGene Technologies, Inc.) for $2 \mathrm{~h}$ at room temperature, the membrane was visualized using an ECL kit (Beyotime Institute of Biotechnology) using a full-automatic chemiluminescence imaging analysis system (Tanon Science and Technology Co., Ltd.). Densitometry was analyzed using ImageJ software (National Institutes of Health).

Subcellular fractionation test. A PARIS kit (Thermo Fisher Scientific, Inc.) was used to separate RNAs in the cytoplasmic and nuclear fractions. RT-qPCR was performed as aforementioned, with U6 and GAPDH as the nuclear and cytoplasmic controls, respectively.

RNA immunoprecipitation (RIP). RIP was conducted using the Magna RIP RNA-Binding Protein immunoprecipitation kit (EMD Millipore) in accordance with the manufacturer's protocols. After transfection for $48 \mathrm{~h}$, GM cells were lysed with RIP lysis buffer. Afterward, cell lysates were incubated with magnetic beads conjugated with anti-Ago2 (1:50; cat. no. ab186733; Abcam), or anti-IgG at $4^{\circ} \mathrm{C}$ for $8 \mathrm{~h}$. The beads were then washed and incubated with Proteinase $\mathrm{K}$ to remove the proteins. After purification, the enrichment of circ-TOP2A was tested using RT-qPCR.

RNA pulldown assay. The biotin-labeled circ-TOP2A probe targeting the junction sequence of circ-TOP2A and oligonucleotide probes (500 pmol) were designed and synthesized in vitro by Wuhan GeneCreate Biological Engineering Co., Ltd. and used for incubation with cell lysates at $4{ }^{\circ} \mathrm{C}$ overnight. Then, the complex was incubated with streptavidin-conjugated magnetic beads (Invitrogen; Thermo Fisher Scientific, Inc.) at $22-25^{\circ} \mathrm{C}$ for $2 \mathrm{~h}$. After purification and RNA extraction (TRIzol method) (15), the enrichment of circ-TOP2A and miRs was measured using RT-qPCR.

Dual-luciferase reporter assay. The circular RNA interactome database (v2020-01-30; circinteractome.nia.nih.gov) was used for predicting the miRs potentially interacting with circ-TOP2A (16). The binding relationship of the 3'-untranslated region (UTR) of SUSD2 and miR-346 was predicted by starBase v2.0 (17). The region sequence of circ-TOP2A or SUSD2 3'-UTR that contained the binding site (wt) as well as the mutated region sequence (mut) were amplified and cloned into the pmirGLO luciferase vector (Promega Corporation). 
293 T cells were co-transfected with miR-346 mimics and plasmids containing the 3'-UTRs of wild-type or mutant sequences of the miR binding site in SUSD2 or wild-type or mutant sequences of circ-TOP2A using Lipofectamine 3000 (Invitrogen; Thermo Fisher Scientific, Inc.) in accordance with the manufacturer's instructions. After transfection for $36 \mathrm{~h}$, the relative luciferase signals of the cells were measured using a Dual-Luciferase Reporter Assay System (Promega Corporation). The specific target activity was expressed as the relative activity ratio of firefly luciferase to Renilla luciferase. The experiment was repeated three times independently.

Cell Counting Kit-8 (CCK-8). Cell viability was assessed using the CCK-8 assay (Tiangen Biotech Co., Ltd.) according to the manufacturer's instructions. In brief, the cells were collected and the concentration was adjusted to $2 \times 10^{3}$ cells/well before they were maintained in a cell incubator for $0,24,48,72$ and $96 \mathrm{~h}$. The cells were then seeded in 96 -well plates at 1,500 cells per well. After the indicated specific treatments, $10 \mu \mathrm{l}$ of CCK-8 was supplied to the wells and maintained at $37^{\circ} \mathrm{C}$ for $2 \mathrm{~h}$. Then, the absorbance of each well at $450 \mathrm{~nm}$ was tested using a microplate reader (Thermo Fisher Scientific, Inc.).

Colony forming test. LN229 and U87MG cells were plated into 6-well plates (1,000 cells/well) with medium containing $10 \% \mathrm{FBS}$ and incubated at $37^{\circ} \mathrm{C}$. After $\sim 10$ days, the colonies were fixed with $4 \%$ paraformaldehyde for $20 \mathrm{~min}$ at room temperature and stained with crystal violet solution (Sigma-Aldrich; Merck KGaA) for another $20 \mathrm{~min}$ at room temperature. Finally, images were captured of the colonies and they were counted manually.

Apoptosis detection. Cell apoptosis assays were performed by flow cytometry (Applied Biosystems; Thermo Fisher Scientific, Inc.). First, GM cells were seeded in 6-well plates before collection by centrifuging at $1,000 \mathrm{x} g$ for $3 \mathrm{~min}$ at room temperature. The cells were washed twice with precooled PBS and the concentration was adjusted to $5 \times 10^{5}-5 \times 10^{6}$ cells with $400 \mu \mathrm{l}$ of staining buffer $(10 \mathrm{mM}$ Hepes/ $\mathrm{NaOH}$, $\mathrm{pH} 7.4 ; 140 \mathrm{mM} \mathrm{NaCl}$ and $2.5 \mathrm{mM} \mathrm{CaCl} 2$ ) containing $5 \mu \mathrm{l}$ of Annexin $\mathrm{V}$ labeled with fluorescein isothiocyanate and $5 \mu \mathrm{l}$ of PI (Annexin V-FITC apoptosis kit; BD Biosciences). Following a 20 -minute incubation at $22-25^{\circ} \mathrm{C}$ in the dark, the treated cells were subjected to apoptosis assays by flow cytometry (FACScan; BD Biosciences). FlowJo v10 software (Tree Star, Inc.) was used for apoptosis analysis. The percentage of early + late apoptotic cells was calculated as the apoptotic rate.

Transwell experiments. A Transwell plate containing an $8-\mu \mathrm{m}$ pore size filter (BD Biosciences) was used to detect the invasion and migration of cells. The cells were suspended in RPMI-1640 with $0.1 \%$ FBS and supplied to the top compartment at a density of 10,000 cells per well. For the cell invasion assay, Matrigel was pre-cooled at $4^{\circ} \mathrm{C}$ overnight and coated on the upper side of the membrane. Then, the chambers were placed in a 24-well plate and $600 \mu \mathrm{l}$ of medium containing $10 \%$ FBS was added to each well. After incubation in a cell incubator at $37^{\circ} \mathrm{C}$ for $24 \mathrm{~h}$, the cells on the upper side of the membrane were removed with cotton swabs and the migrated/invaded cells in the lower membrane of the chambers were fixed by paraformaldehyde at room temperature for $20 \mathrm{~min}$ and then stained with crystal violet for $20 \mathrm{~min}$ at room temperature. Images were captured from five randomly selected fields of view (magnification, x200) under a light microscope.

Data analysis. All results are presented as the mean \pm standard deviation and were analyzed with GraphPad Prism 8.0 (GraphPad Software, Inc.) and SPSS 22.0 (IBM Corp.). Unpaired Student's t test was used to compare the significance of differences between two groups. For comparisons between cancerous and adjacent normal tissues, paired t test was used. One-way ANOVA with post hoc Tukey's test was used to compare the significance of differences among three or more groups. Kaplan-Meier analysis with log-rank test was applied to measure the overall survival rate (from surgery to death) and progression-free survival (from surgery to tumor recurrence/metastasis) of GM patients. The Cancer Genome Atlas (TCGA) and Pearson's correlation analysis was used to assess the correlation between SUSD2 and miR-346 expression levels, as well as patient survival data. $\mathrm{P}<0.05$ was considered to indicate a statistically significant difference.

\section{Results}

Circ-TOP2A is overexpressed in GM and is associated with poor prognosis. A total of five circRNAs, circ-CLSPN, circ-TOP2A, circ-MELK, circ-BUB1 and circ-KIF4A, were selected from the sequencing data (12). Total RNA was isolated from 10 pairs of GM and normal tissues to detect the expression of circRNAs. As shown in Fig. 1B, circ-TOP2A was the most upregulated circRNA expressed in GM tissues compared with its the normal counterparts. Therefore, it was chosen for further study. Circ-TOP2A (circ_0043548) was mapped to chr17:38556460-38569223. It was spliced from exons 7-23 of TOP $2 A$. The spliced variant of circ-TOP2A was 2424 nucleotides long (Fig. 1C). It was determined that circ-TOP2A was more stable than TOP2A mRNA (Fig. 1D). In addition, total RNA was extracted to detect the expression of circ-TOP2A and linear TOP2A mRNA following treatment with actinomycin $\mathrm{D}$ at different time points. Linear TOP2A showed a shorter half-life compared with circ-TOP2A (Fig. 1E). RT-qPCR demonstrated that circ-TOP2A was markedly elevated in GM specimens compared with noncancerous samples (Fig. 1F). To explore the prognostic value of circ-TOP2A, patients were divided into high- and low-circ-TOP2A groups according to the median expression level of circ-TOP2A in cancer tissues. As illustrated in Fig. $1 \mathrm{G}$ and $\mathrm{H}$, Kaplan-Meier curves revealed that overall survival rate $\left(\mathrm{P}=3.1 \times 10^{-2}\right)$ and progression-free survival $\left(\mathrm{P}=3.5 \times 10^{-2}\right)$ were lower in the high-circ-TOP2A group compared with the low-circ-TOP2A group. Furthermore, the expression of circ-TOP2A was found to be overexpressed in GM cell lines compared with NHA cells (Fig. 1I).

Circ-TOP $2 A$ promotes $G M$ cell proliferation and aggressiveness. The cellular function of circ-TOP2A by down-/up-regulation of circ-TOP2A was then examined in GM cell lines. LN229 cells were used for the knockdown study due to their high expression of circ-TOP2A. The silencing efficiencies of si-circ-TOP2A-1 and si-circ-TOP2A-2 were 

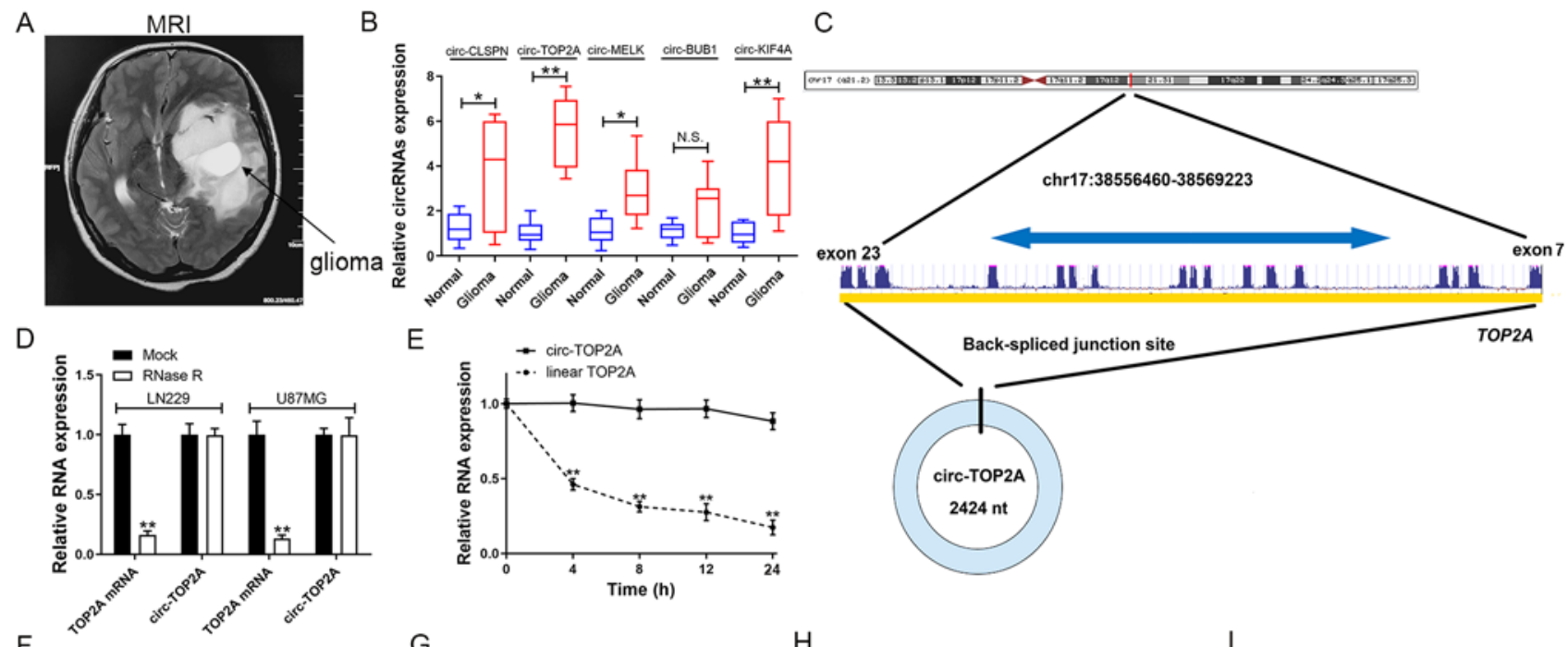

E
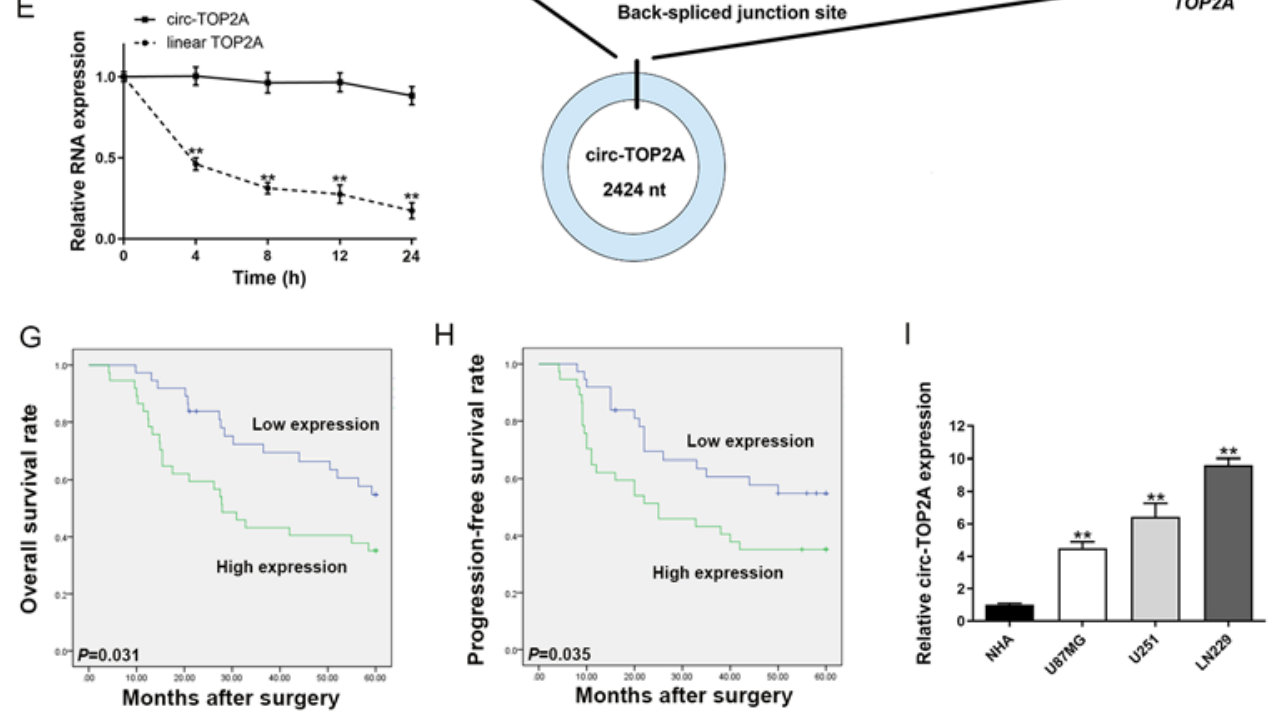

Figure 1. Circ-TOP2A expression in GM tissues and cells and its clinical importance. (A) Representative image of GM by magnetic resonance imaging. (B) Circ-CLSPN, circ-TOP2A, circ-MELK, circ-BUB1 and circ-KIF4A expression was evaluated by RT-qPCR in GM tissues/adjacent normal tissues (C) Schematic representation of circ-TOP2A formation. (D) Circ-TOP2A was resistant to RNase R digestion in GM cells. (E) Relative circ-TOP2A and linear TOP2A mRNA expression at different time points. (F) Circ-TOP2A expression in 74 pairs of GM tissues/adjacent normal tissues by RT-qPCR. (G) Kaplan-Meier analysis with log-rank test for overall survival in GM patients according to circ-TOP2A expression. (H) Kaplan-Meier analysis with log-rank test for progression-free survival in GM patients according to circ-TOP2A expression. (I) Relative expression of circ-TOP2A in GM and normal cells by RT-qPCR. D, $\mathrm{E}$ and I: The data are shown as the mean \pm standard deviation $(\mathrm{n}=3)$. ${ }^{*} \mathrm{P}<0.05,{ }^{* *} \mathrm{P}<0.01$. Circ, circRNA; GM, glioma; MRI, magnetic resonance imaging; RT-qPCR, reverse transcription-quantitative PCR; NHA, normal human astrocytes.

favorable (Fig. 2A). Circ-TOP2A expression was ectopically expressed in U87MG cells due to its lowest level of circ-TOP2A among the enrolled GM cell lines (Fig. 2A). As presented in Fig. 2B, transfection with circ-TOP2A siRNAs or vector could not down-/up-regulate TOP2A mRNA expression levels. Functionally, silencing of circ-TOP2A significantly impeded cell viability and clone-forming ability in LN229 cells (Fig. 2C and E). Overexpression of circ-TOP2A caused the opposite effect in U87MG cells (Fig. 2D and F). Additionally, down and upregulation of circ-TOP2A respectively triggered or inhibited cell apoptosis in GM cells (Fig. 2G and $\mathrm{H}$ ). In addition, circ-TOP2A downregulation suppressed cell migration and invasion in LN229 cells (Fig. 2I). In contrast, overexpression of circ-TOP2A exerted the opposite effect in U87MG cells (Fig. 2J).

Circ-TOP2A elevates SUSD2 expression by sponging miR-346 in GM cells. RNA-Seq was conducted and generated a heatmap for the 10 most differentially expressed mRNAs in circ-TOP2A-knockdown and control cells. SUSD2 was a common gene target of circ-TOP2A in LN229 and U87MG cells (Fig. 3A and B). Additionally, RT-qPCR validated that circ-TOP2A could positively regulate SUSD2 expression levels (Fig. 3C). Furthermore, a subcellular distribution assay illustrated that circ-TOP2A was primarily localized in the cytoplasm of GM cells (Fig. 3D). The starBase v2.0 database predicted $72 \mathrm{miRs}$ that potentially bind with SUSD2 3'-UTR. The circular RNA interactome database predicted $89 \mathrm{miRs}$ that may be sponged by circ-TOP2A. miR-217, miR-383-5p, miR-23c, miR-346, miR-494-3p, miR-23b-3p, miR-873-5p and miR-1286 were identified as common miRs in the two databases (Fig. 3E). As shown in Fig. 4A, circ-TOP2A was markedly enriched in the anti-ago 2 immunoprecipitated pool compared with the anti-IgG pool. In addition, knockdown of circ-TOP2A partly weakened this binding ability. Circ-TOP2A was then pulled down using a specific probe in LN229 and U87MG cells. Ectopic expression of circ-TOP2A could enhance this efficiency (Fig. 4B). RNA pulldown assays indicated that only miR-346 could interact with circ-TOP2A in LN229 cells (Fig. 4C). Coefficient correlation analysis uncovered a negative association between SUSD2 mRNA and miR-346 expression $\left(\mathrm{P}=8.0 \times 10^{-3}\right)$. The data also showed that the expression of SUSD2 mRNA was positively linked to circ-TOP2A expression ( $\mathrm{P}=3.0 \times 10^{-3}$; Fig. 4D and E). Similarly, TCGA data showed a negative correlation between SUSD2 mRNA and miR-346 in GM tissue samples $\left(\mathrm{P}=7.19 \times 10^{-11}\right.$; Fig. 4F). Kaplan-Meier curves demonstrated that the patients with high expression of SUSD2 had a worse overall survival rate $\left(\mathrm{P}=6.6 \times 10^{-6}\right.$; Fig. $\left.4 \mathrm{~F}\right)$. Pearson's correlation analysis indicated a negative association between SUSD2 and miR-346 expression levels (Fig. 4G). 
A

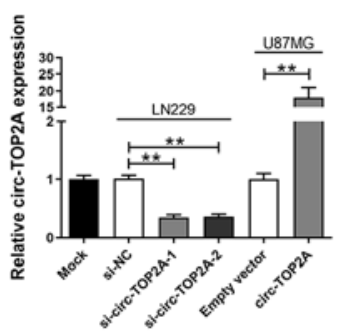

E

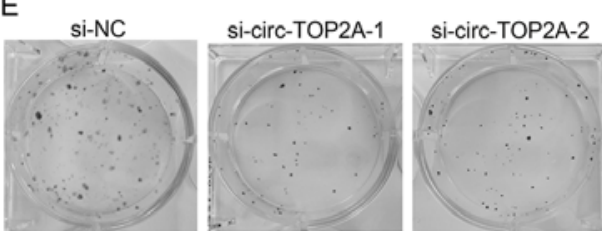

G
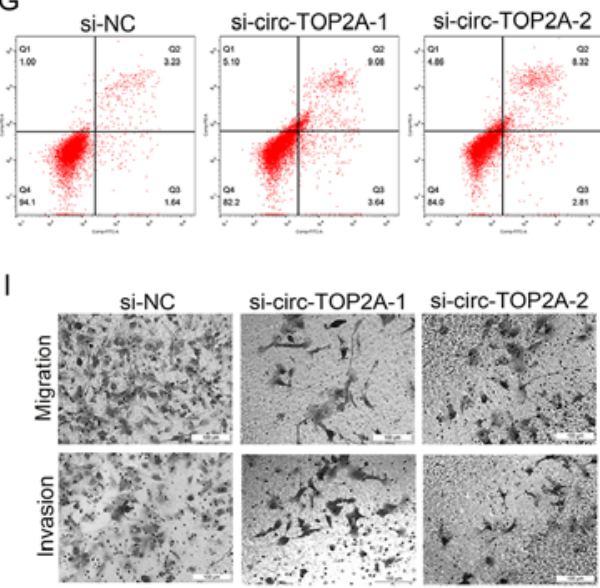

B
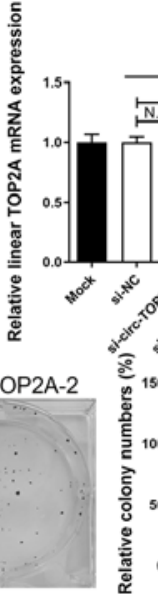

C

LN229

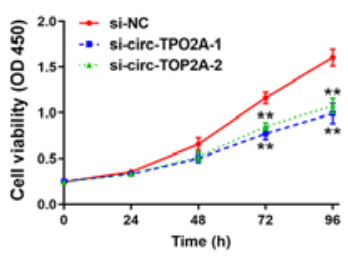

F
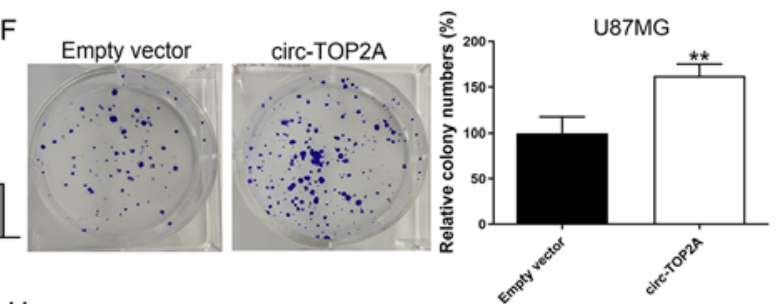

$\mathrm{H}$
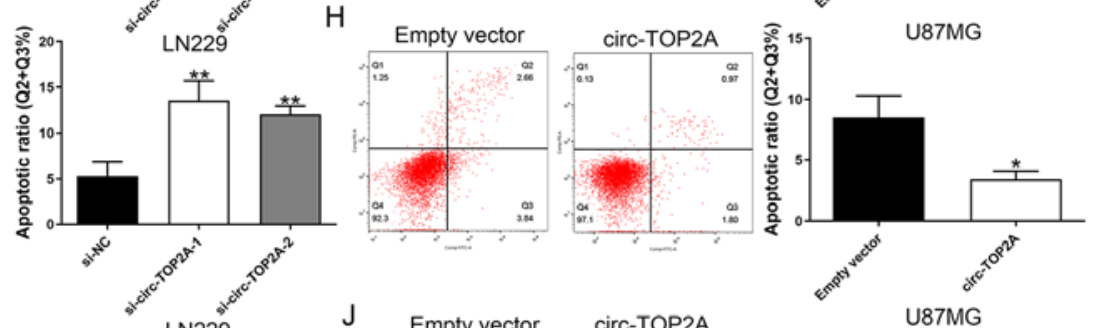

U87MG
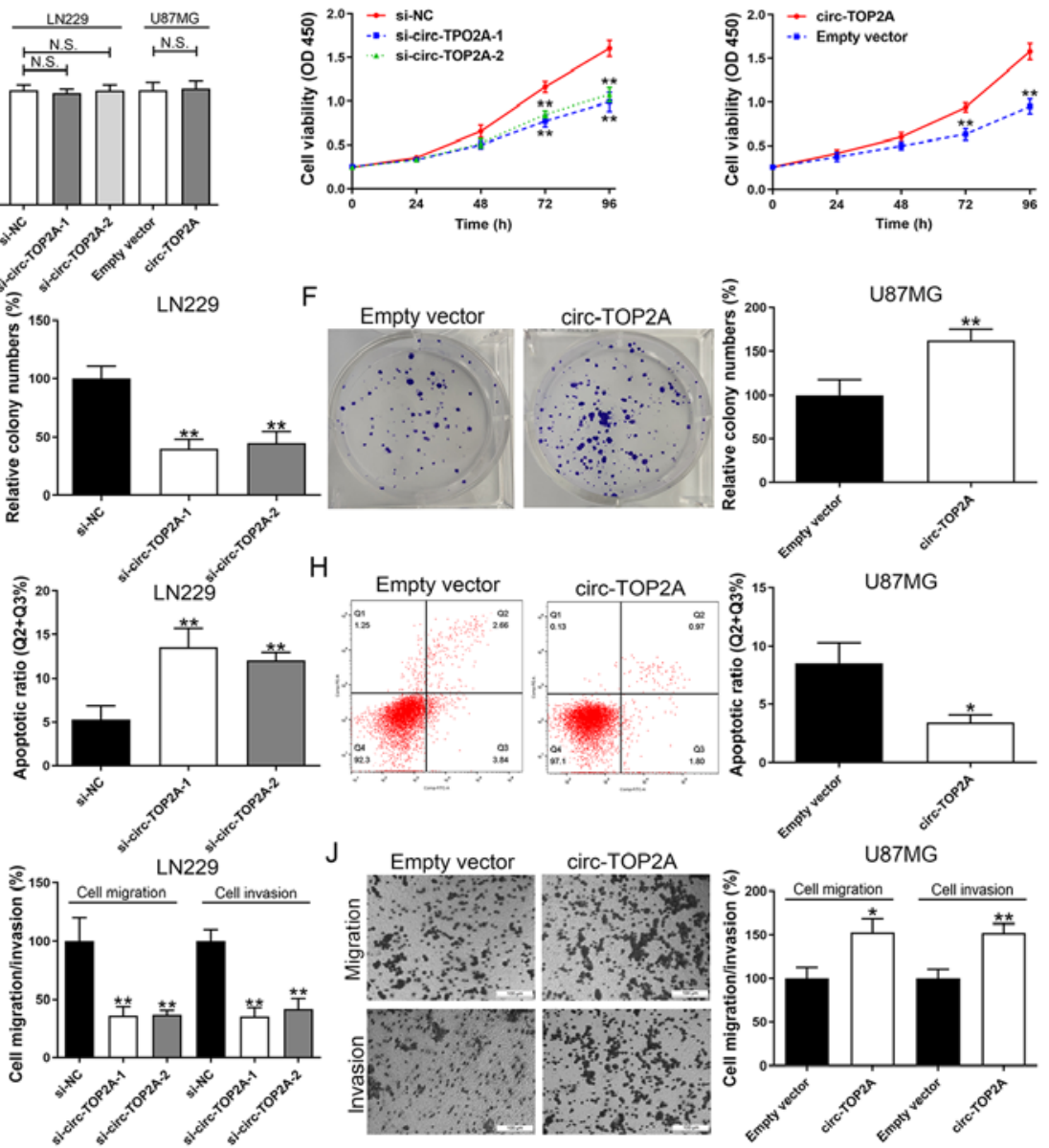

U87MG

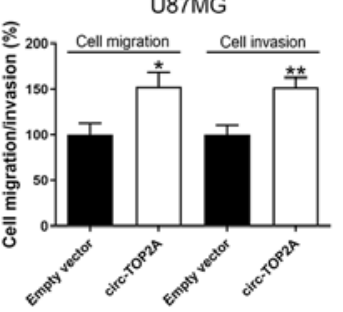

Figure 2. Circ-TOP2A facilitates GM cell progression. (A) Relative expression of circ-TOP2A was detected by RT-qPCR following transfection. (B) Relative expression of TOP2A mRNA was detected by RT-qPCR following transfection. Cell viability was detected after down-/up-regulating circ-TOP2A in (C) LN229 and (D) U87MG cells by CCK-8. Clone-forming ability was detected after down-/up-regulating circ-TOP2A in (E) LN229 and (F) U87MG cells by colony formation assay. Cell apoptosis was detected after down-/up-regulating circ-TOP2A in (G) LN229 and (H) U87MG cells by flow cytometry. Cell migration and invasion was detected after down-/up-regulating circ-TOP2A in (I) LN229 and (J) U87MG cells by Transwell assay. A-J: The data are shown as the mean \pm standard deviation $(\mathrm{n}=3)$. ${ }^{*} \mathrm{P}<0.05,{ }^{* *} \mathrm{P}<0.01$. Circ, circRNA; GM, glioma; RT-qPCR, reverse transcription-quantitative PCR.

Conversely, miR-346 expression in GM tissues correlated with favorable prognosis analyzed by the TCGA dataset $\left(\mathrm{P}=1.1 \times 10^{-2}\right)$. As expected, miR-346 mimics/inhibitor could significantly up/downregulated miR-346 expression (Fig. 4I). It was further confirmed that SUSD2 vector/si-SUSD2 could effectively contribute to SUSD2 increase/decrease (Fig. 4J). In addition, SUSD2 mRNA expression was negatively regulated by miR-346 (Fig. 4J). Furthermore, miR-346 expression in GM cell lines was generally decreased, which is conversely associated with circ-TOP2A (Fig. 1I) and SUSD2 expression levels (Fig. 4K and L). To verify the target binding between circ-TOP2A and miR-346, a dual-luciferase reporter gene test was performed in constructed wild-type and mutant circ-TOP2A (Fig. 4M). As shown in Fig. 4N, miR-346 markedly decreased the luciferase intensity in wild-type circ-TOP2A but had no effect on binding motif mutations. In addition, the binding ability between the 3'-UTR of SUSD2 and miR-346 was further verified by a dual-luciferase reporter gene test (Fig. 4M and N).

Circ-TOP2A/miR-346/SUSD2 signaling is critical for GM cell function. Rescue experiments were performed to reveal the mechanisms of circ-TOP2A in GM. As shown in Fig. 5A, the protein level of SUSD2 was decreased in the si-circ-TOP2A-1 group and changes in SUSD2 expression were reversed by co-transfection with miR-346 inhibitor or SUSD2 vector. In addition, SUSD2 expression was elevated following transfection with the circ-TOP2A-overexpressing vector in U87MG cells. After co-transfection with miR-346 mimics or si-SUSD2, the protein expression of SUSD2 was partly inhibited (Fig. 5A). Additionally, relative expression of circ-TOP2A was decreased following transfection with si-circ-TOP2A-1. Si-circ-TOP2A-1 co-transfection with miR-346 inhibitor or SUSD2 vector did not alter circ-TOP2A expression in LN229 cells. Similarly, circ-TOP2A vector led to increased expression of circ-TOP2A. Further co-transfection with miR-346 mimics or si-SUSD2 had no effect on circ-TOP2A expression in U87MG cells (Fig. S1A). miR-346 expression was unchanged after knockdown or overexpression of circ-TOP2A, which implied that circ-TOP2A sponges miR-346 to inhibit its functions rather than expression levels. Co-transfection with miR-346 inhibitor and miR-346 mimics markedly decreased and increased miR-346 expression, respectively (Fig. S1B). 
A

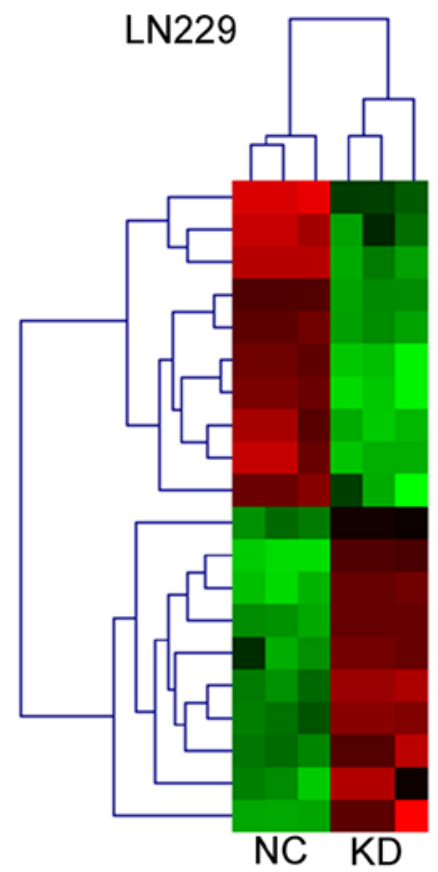

C

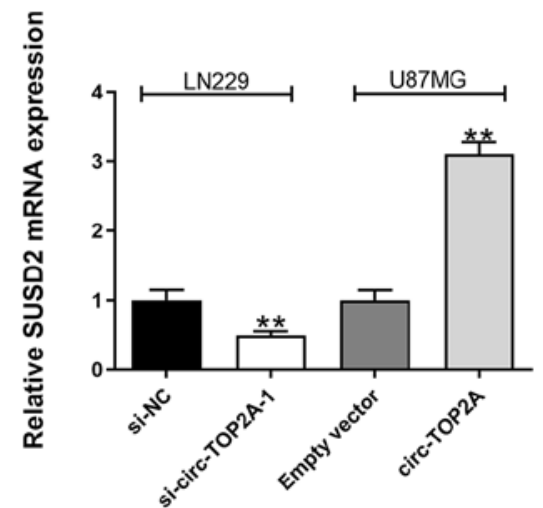

$\mathrm{E}$

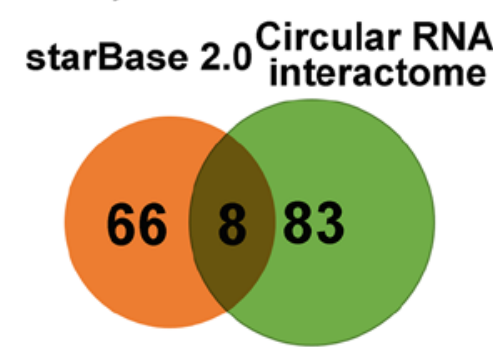

B
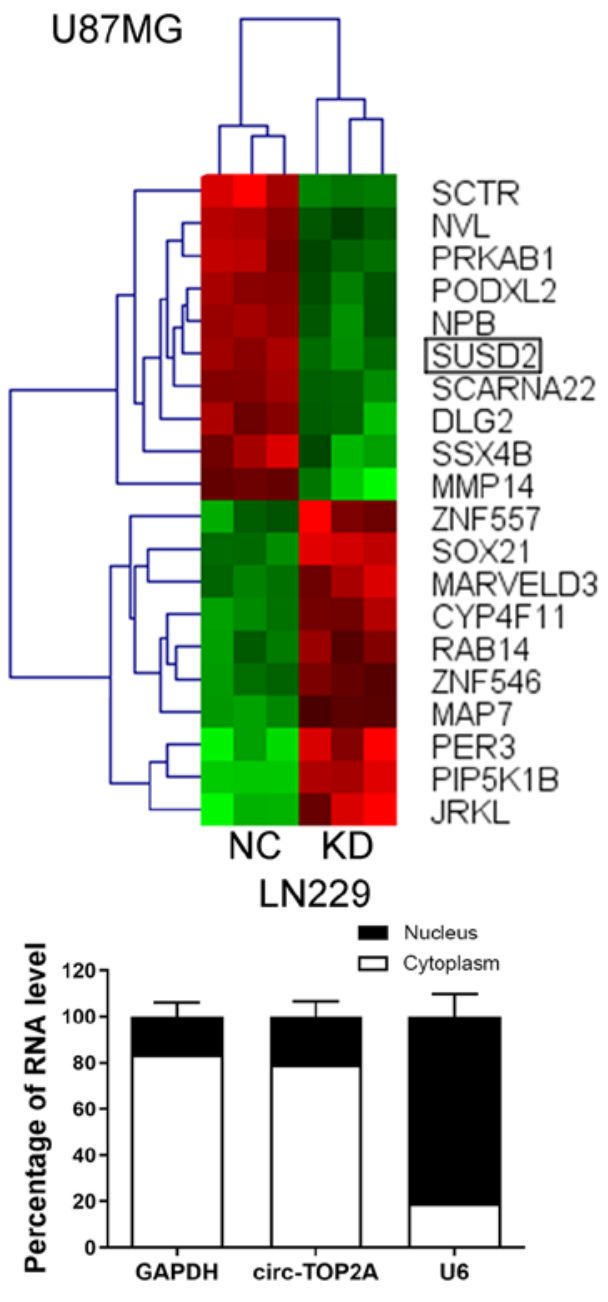

U87MG

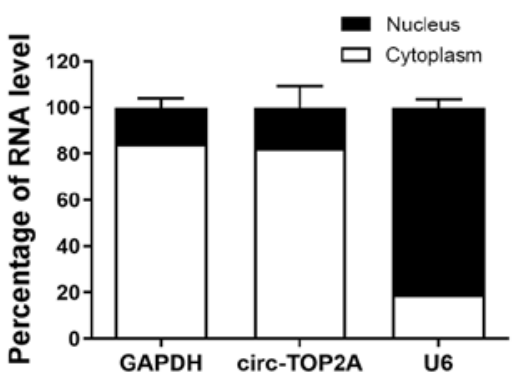

Figure 3. SUSD2 is the potential target of circ-TOP2A. Clustered heatmap showing circ-TOP2A-regulated mRNAs in (A) LN229 and (B) U87MG cells. (C) SUSD2 mRNA expression was detected after down-/up-regulating circ-TOP2A in LN229 and U87MG cells by RT-qPCR. (D) RT-qPCR detection of the percentage of circ-TOP2A in the cytoplasmic and nuclear fractions of LN229 and U87MG cells. (E) Venn diagram showing the number of overlapping miRs. C-D: The data are shown as the mean \pm standard deviation $(n=3) .{ }^{* *} \mathrm{P}<0.01$. Circ, circRNA; RT-qPCR, reverse transcription-quantitative PCR; si, short interfering; $\mathrm{NC}$, negative control.

CCK-8, colony formation and Transwell assays indicated that the inhibitory effects of si-circ-TOP2A on cell proliferation and invasion were attenuated by co-transfection with miR-346 inhibitor or SUSD2 vector (Fig. 5B, D and F). Overexpression of the circ-TOP2A vector led to increased cell viability, clone-forming ability and invasive potential in U87MG cells. These malignant behaviors were partially rescued after co-transfection with miR-346 mimics or si-SUSD2 (Fig. 5C, E and G). Taken together, these findings revealed that the circ-TOP2A mediated miR-346/SUSD2 axis triggered GM carcinogenesis.

\section{Discussion}

Tumorigenesis and metastasis are complicated processes involving the activation of oncogenes and the inactivation of tumor suppressor genes. CircRNAs are reported to regulate gene expression at epigenetic, transcriptional and posttranscriptional levels, mediating the initiation and metastasis of tumors $(10,18,19)$. Circ-TOP2A was screened as an upregulated circRNA in GM tissue samples compared with noncancerous tissues (12). The current study further identified that the expression of circ-TOP2A was upregulated in GM tissues and cell 

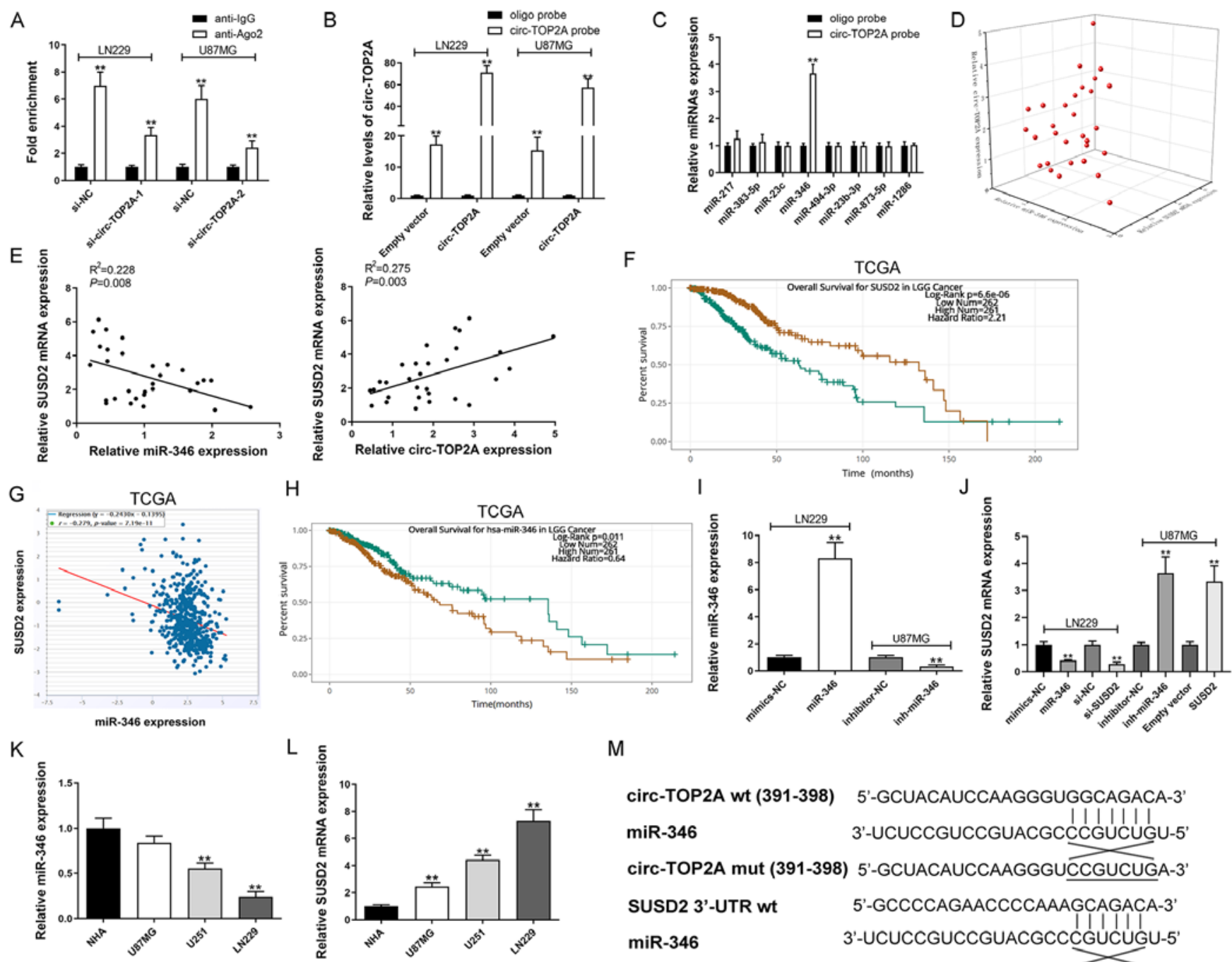

M

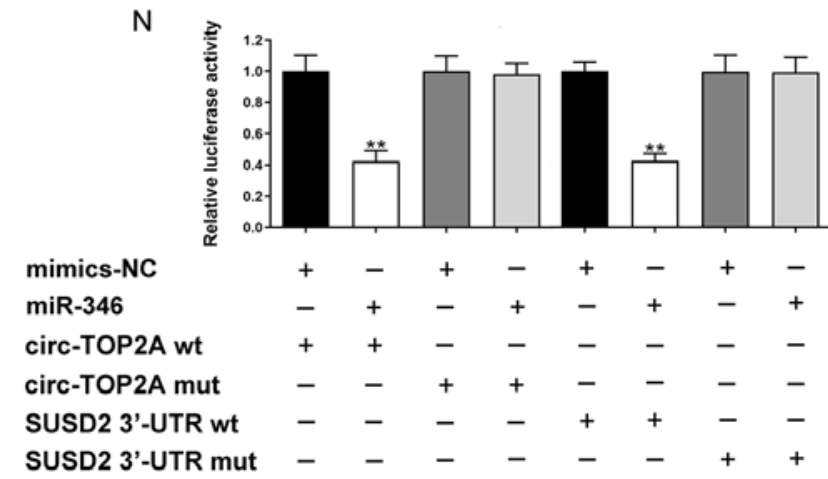

Figure 4. Circ-TOP2A sponges miR-346 to upregulate SUSD2 expression in GM. (A) Ago2-RNA immunoprecipitation assay for circ-TOP2A levels in LN229 and U87MG cells following transfection. (B) Lysates prepared from LN229 and U87MG cells following transfection were subjected to RNA pull-down assay. (C) RT-qPCR for miR-217, miR-383-5p, miR-23c, miR-346, miR-494-3p, miR-23b-3p, miR-873-5p and miR-1286 expression in LN229 cell lysates. (D and E) Correlation among circ-TOP2A, miR-346 and SUSD2 mRNA in GM samples. (F) Kaplan-Meier analysis of overall survival in GM patients according to SUSD2 expression by TCGA data. (G) Correlation analysis of SUSD2 and miR-346 expression in GM/normal tissues by TCGA data. (H) Kaplan-Meier analysis of overall survival in GM patients according to miR-346 expression by TCGA data. (I) miR-346 expression was detected by RT-qPCR after down-/up-regulating miR-346 in LN229 and U87MG cells. (J) SUSD2 mRNA expression was detected by RT-qPCR following transfection in LN229 and U87MG cells. (K) Relative expression of miR-346 in GM and normal cells by RT-qPCR. (L) Relative expression of SUSD2 mRNA in GM and normal cells by RT-qPCR. (M) Schematic illustration of circ-TOP2A-wt/mut and SUSD2 3'-UTR-wt/mut luciferase reporter vectors. (N) The binding ability between circ-TOP2A/SUSD2 3'-UTR and miR-346 was detected by dual-luciferase reporter assay in 293T cells. A-C, I-L and N: The data are shown as the mean \pm standard deviation $(\mathrm{n}=3) .{ }^{* * *} \mathrm{P}<0.01$. Circ, circRNA; RT-qPCR, reverse transcription-quantitative PCR; miR, microRNA; GM, glioma; wt, wild-type; mut, mutant; TCGA, The Cancer Genome Atlas; UTR, untranslated region; NC, negative control.

lines (LN229, U251 and U87MG). The results were obtained based on $74 \mathrm{GM}$ tissue samples. It was identified that high expression of circ-TOP2A in GM specimens correlated with adverse prognosis and high progression-free survival. However, due to the limited number of patients recruited, the independent prognostic role of circ-TOP2A was not explored in this study. 


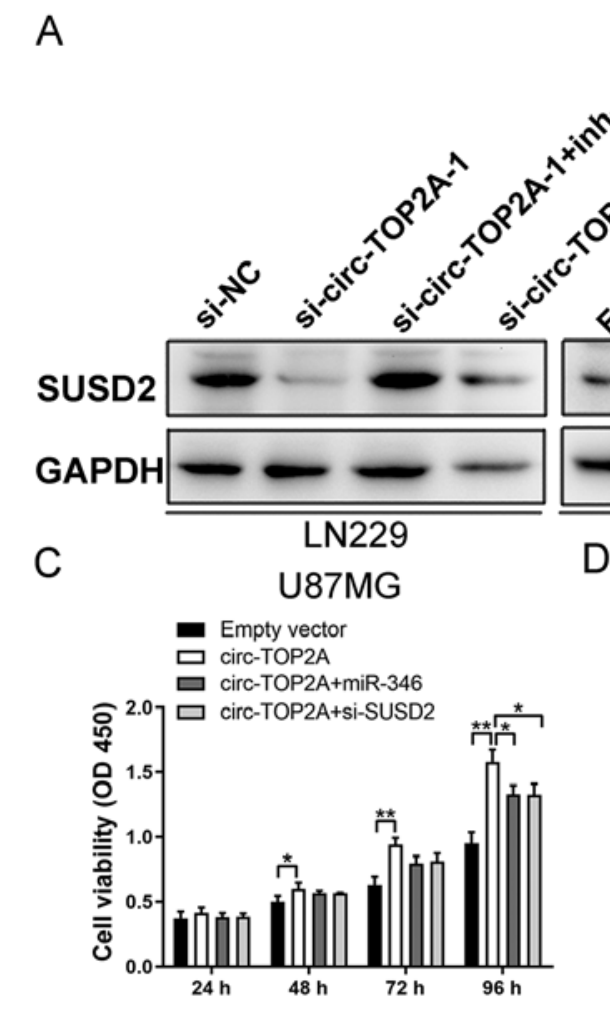

B
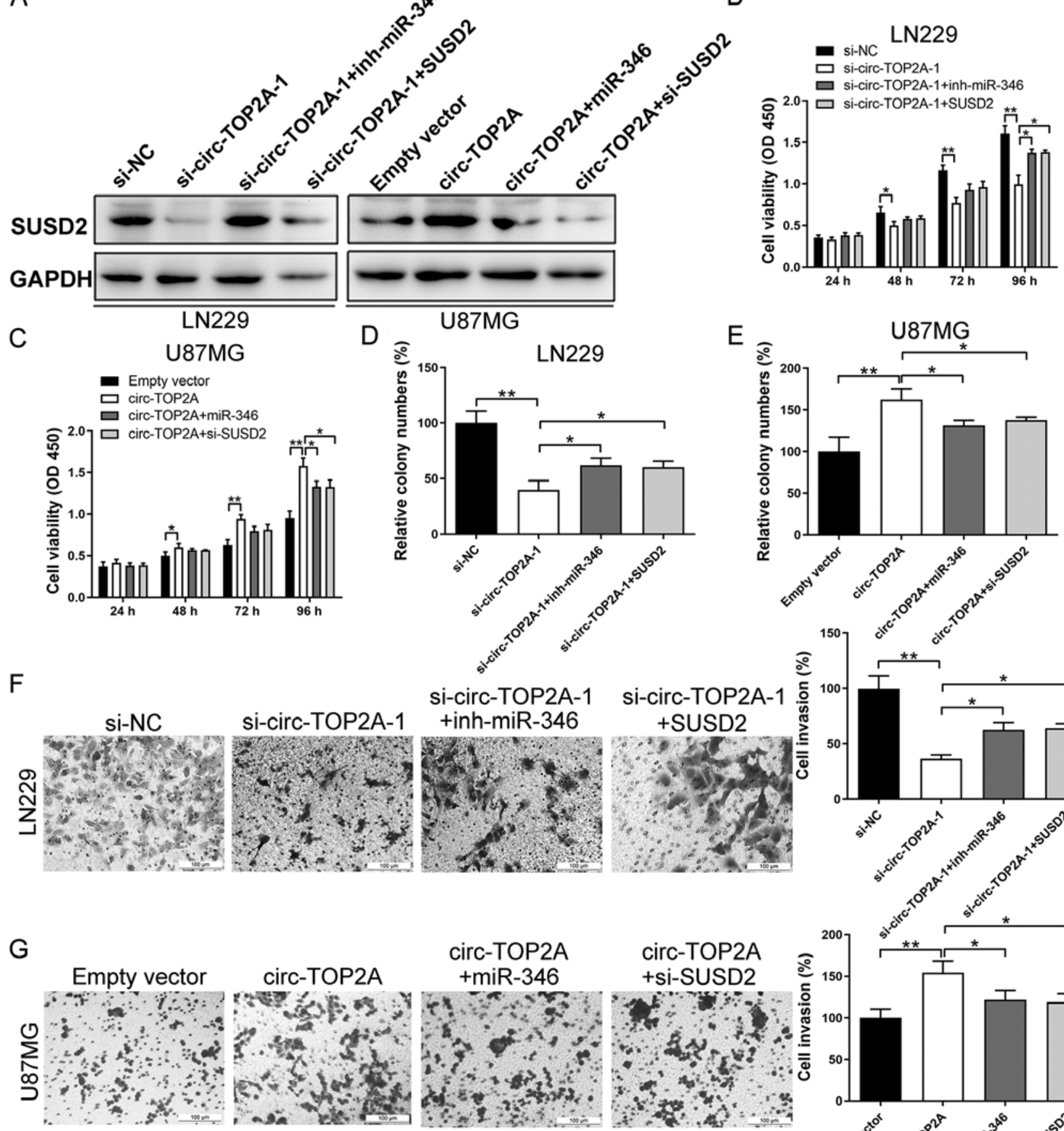

E

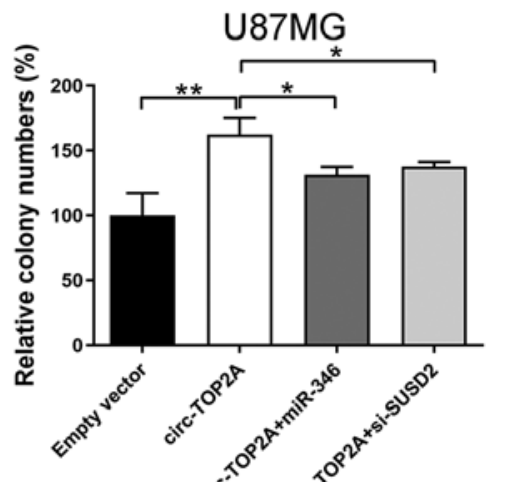


as a novel target of circ-TOP2A. miR-346 is reported to target and inhibit the expression of a number of classical oncogenes and then suppress the progression of a number of cancers $(22,23)$. Previously, miR-346 was reported as a tumor suppressor in GM by directly targeting NFIB (24). The present study validated the tumor suppressive function of miR-346 in GM and found that a miR-346 inhibitor could reverse the anticancer effects of circ-TOP2A knockdown. These results demonstrated that circ-TOP2A facilitates GM growth and aggressiveness, at least in part via repression of the function of miR-346. SUSD2, a type I transmembrane protein that localizes to the cell surface, has been reported to be upregulated in a number of tumors and to promote cancer progression and metastasis $(25,26)$. However, some studies have indicated that upregulated SUSD2 impedes cell progression in several malignancies, suggesting a possible tumor suppressive role of SUSD2 $(27,28)$. These previous studies indicate that SUSD2 might have a complex function in different malignancies. Consistent with the studies on breast cancer and ovarian cancer $(25,26)$, SUSD2 was obviously increased in GM tissues and cells compared with matched control groups. Notably, the present study also demonstrated that overexpression of SUSD2 acted as a tumorigenic function in circ-TOP2A-downregulated GM cells, suggesting the cancerogenic function of SUSD2 in GM. Collectively, circ-TOP2A promoted GM proliferation and aggressiveness through the miR-346/SUSD2 signaling pathway.

In conclusion, the results of the present study suggested that circ-TOP2A was increased, circular and stable in GM cells. Notably, upregulation of circ-TOP2A exerted its functions by facilitating cell growth, migration and invasion, while inhibiting apoptosis in GM cells by targeting miR-346/SUSD2 signaling. Therefore, circ-TOP $2 \mathrm{~A}$ may be a potential prognostic biomarker/therapeutic target for patients with GM.

\section{Acknowledgements}

Not applicable.

\section{Funding}

No funding was received.

\section{Availability of data and materials}

All data generated or analyzed during this study are included in this published article.

\section{Authors' contributions}

$\mathrm{CZ}, \mathrm{XZ}$ and GL analyzed and interpreted patient data regarding the hematological disease and transplant. JS, XL and LL performed the in vitro assay. JS wrote the manuscript. JS and XL confirmed the authenticity of raw data. All authors read and approved the final manuscript.

\section{Ethics approval and consent to participate}

The research was authorized by the Ethics Committee of The Second Affiliated Hospital of Qiqihar Medical University (approval number: KY2020-012) and written informed consent was acquired from each patient.

\section{Patient consent for publication}

Not applicable.

\section{Competing interests}

The authors declare that they have no competing interests.

\section{References}

1. Farina P, Lombardi G, Bergo E, Roma A and Zagonel V: Treatment of malignant gliomas in elderly patients: A concise overview of the literature. Biomed Res Int 2014: 734281, 2014.

2. Wen PY and Huse JT: 2016 World Health Organization classification of central nervous system tumors. Continuum (Minneap Minn) 23: 1531-1547, 2017.

3. Omuro A and DeAngelis LM: Glioblastoma and other malignant gliomas: A clinical review. JAMA 310: 1842-1850, 2013.

4. Gusyatiner O and Hegi ME: Glioma epigenetics: From subclassification to novel treatment options. Semin Cancer Biol 51: 50-58, 2018.

5. Salzman J: Circular RNA expression: Its potential regulation and function. Trends Genet 32: 309-316, 2016.

6. Eger N, Schoppe L, Schuster S, Laufs U and Boeckel JN: Circular RNA Splicing. Adv Exp Med Biol 1087: 41-52, 2018.

7. Sanger HL, Klotz G, Riesner D, Gross HJ and Kleinschmidt AK: Viroids are single-stranded covalently closed circular RNA molecules existing as highly base-paired rod-like structures. Proc Natl Acad Sci USA 73: 3852-3856, 1976.

8. Han B, Chao J and Yao H: Circular RNA and its mechanisms in disease: From the bench to the clinic. Pharmacol Ther 187: 31-44, 2018

9. Belousova EA, Filipenko ML and Kushlinskii NE: Circular RNA: New regulatory molecules. Bull Exp Biol Med 164: 803-815, 2018.

10. Chen B and Huang S: Circular RNA: An emerging non-coding RNA as a regulator and biomarker in cancer. Cancer Lett 418: 41-50, 2018

11. Xu Y, Yao Y, Zhong X, Leng K, Qin W, Qu L, Cui Y and Jiang X: Downregulated circular RNA hsa_circ_0001649 regulates proliferation, migration and invasion in cholangiocarcinoma cells. Biochem Biophys Res Commun 496: 455-461, 2018.

12. Wang R, Zhang S, Chen X, Li N, Li J, Jia R, Pan Y and Liang H: EIF4A3-induced circular RNA MMP9 (circMMP9) acts as a sponge of miR-124 and promotes glioblastoma multiforme cell tumorigenesis. Mol Cancer 17: 166, 2018.

13. Hu H, Wang Y, Zhang T, Zhang C, Liu Y, Li G, Zhou D and Lu S: Association of LncRNA-GACAT3 with MRI features of breast cancer and its molecular mechanism. J BUON 24: 2377-2384, 2019.

14. Schmittgen TD and Livak KJ: Analyzing real-time PCR data by the comparative C(T) method. Nat Protoc 3: 1101-1108, 2008.

15. Mannhalter C, Koizar D and Mitterbauer G: Evaluation of RNA isolation methods and reference genes for RT-PCR analyses of rare target RNA. Clin Chem Lab Med 38: 171-177, 2000.

16. Dudekula DB, Panda AC, Grammatikakis I, De S, Abdelmohsen K and Gorospe M: CircInteractome: A web tool for exploring circular RNAs and their interacting proteins and microRNAs. RNA Biol 13: 34-42, 2016.

17. Li JH, Liu S, Zhou H, Qu LH and Yang JH: starBase v2.0: Decoding miRNA-ceRNA, miRNA-ncRNA and protein-RNA interaction networks from large-scale CLIP-Seq data. Nucleic Acids Res 42: D92-D97, 2014.

18. Xu Y, Yao Y, Leng K, Ji D, Qu L, Liu Y and Cui Y: Increased expression of circular RNA circ_0005230 indicates dismal prognosis in breast cancer and regulates cell proliferation and invasion via miR-618/CBX8 signal pathway. Cell Physiol Biochem 51: 1710-1722, 2018.

19. Xu Y, Yao Y, Gao P and Cui Y: Upregulated circular RNA circ_0030235 predicts unfavorable prognosis in pancreatic ductal adenocarcinoma and facilitates cell progression by sponging miR-1253 and miR-1294. Biochem Biophys Res Commun 509: 138-142, 2019. 
20. Qi X, Zhang DH, Wu N, Xiao JH, Wang X and Ma W: ceRNA in cancer: Possible functions and clinical implications. J Med Genet 52: 710-718, 2015.

21. Wang Q, Shi L, Shi K, Yuan B, Cao G, Kong C, Fu J, Man Z, Li X, Zhang X, et al: CircCSPP1 functions as a ceRNA to promote colorectal carcinoma cell EMT and liver metastasis by upregulating COL1A1. Front Oncol 10: 850, 2020.

22. Bai N, Peng E, Qiu X, Lyu N, Zhang Z, Tao Y, Li X and Wang Z: circFBLIM1 act as a ceRNA to promote hepatocellular cancer progression by sponging miR-346. J Exp Clin Cancer Res 37: $172,2018$.

23. Zhu W, Qian J, Ma L, Ma P, Yang F and Shu Y: MiR-346 suppresses cell proliferation through SMYD3 dependent approach in hepatocellular carcinoma. Oncotarget 8: 65218-65229, 2017.

24. Li Y, Xu J, Zhang J, Zhang J, Zhang J and Lu X: MicroRNA-346 inhibits the growth of glioma by directly targeting NFIB. Cancer Cell Int 19: 294, 2019.

25. Hultgren EM, Patrick ME, Evans RL, Stoos CT and Egland KA SUSD2 promotes tumor-associated macrophage recruitment by increasing levels of MCP-1 in breast cancer. PLoS One 12 : e0177089, 2017.
26. Xu Y, Miao C, Jin C, Qiu C, Li Y, Sun X, Gao M, Lu N and Kong B: SUSD2 promotes cancer metastasis and confers cisplatin resistance in high grade serous ovarian cancer. Exp Cell Res 363: 160-170, 2018.

27. Cheng Y, Wang X, Wang P, Li T, Hu F, Liu Q, Yang F, Wang J, $\mathrm{Xu} \mathrm{T}$ and Han W: SUSD2 is frequently downregulated and functions as a tumor suppressor in RCC and lung cancer. Tumour Biol 37: 9919-9930, 2016

28. Sheets JN, Patrick ME and Egland KA: SUSD2 expression correlates with decreased metastasis and increased survival in a high-grade serous ovarian cancer xenograft murine model. Oncotarget 11: 2290-2301, 2020.

This work is licensed under a Creative Commons Attribution-NonCommercial-NoDerivatives 4.0 International (CC BY-NC-ND 4.0) License. 